\title{
FUNCIONES PRAGMÁTICAS PRIMARIAS DE LOS GERUNDIOS EN ESPAÑOL INFANTIL
}

\section{PRIMARY PRAGMATIC FUNCTIONS IN CHILD SPANISH GERUNDS}


El uso pragmático atañe directamente el interés de los niños por la lengua, ya que esta les permite hacer cosas con ella e influir en su mundo. Los gerundios refieren al aspecto imperfectivo durativo y en este sentido están en competencia con el presente indicativo, lo que les confiere una función muy específica dentro de la lengua. ¿Cuál es esta función en el habla infantil temprana? Para contestar a esta pregunta se revisaron las estructuras enunciativas con gerundio de dos niñas y un niño en la base de datos ETAL-UNAM (Rojas Nieto, 2007) en un corte longitudinal en interacción espontánea (aprox 1;10-4;00). Los resultados sugieren que dentro del universo lingüístico del español su función pragmática primaria es la función asertiva de la descripción, que sin perder su naturaleza, toma posteriormente también funciones concomitantes a ésta.

Palabras Clave: adquisición de la lengua materna, gerundios, pragmática, actos de habla

Pragmatic use is directly linked to the child's interest for language since it allows her to do things with words and influence her world. Spanish Gerunds make reference to a durative imperfective aspect which is in competence with present indicative, this competence confers Spanish Gerunds an specific function in language. Which is this function in Child Spanish Speech? In order to answer this question all of the sentences with Gerund were analyzed in the ETAL-UNAM Database (Rojas Nieto 2007), a longitudinal sample (1;10-4;00 aprox.) of three children in spontaneous interaction was chosen for this purpose. According to the results obtained Gerunds Primary Pragmatic function is assertive, since pure description are their basic usage goal which take slowly additional functions.

Key words: Child Language Acquisition, Gerunds, Pragmatics, Speech Acts

FECHA DE RECEPCIÓN: 26/08/2015

FECHA DE ACEPTACiÓN: 10/11/2015 


\title{
FUNCIONES PRAGMÁTICAS PRIMARIAS DE LOS GERUNDIOS EN ESPAÑOL INFANTIL
}

\section{PRIMARY PRAGMATIC FUNCTIONS IN CHILD SPANISH GERUNDS}

\author{
Mary R. Espinosa Ochoa \\ Universidad Nacional Autónoma de México
}

La relevancia de la pragmática en el estudio de la adquisición del lenguaje

En la literatura sobre adquisición de lengua materna existe un creciente interés por el campo de la pragmática que permite vislumbrar una visión más completa del proceso frente a los estudios que se han enfocado únicamente en la gramática como forma aislada. Un infante está por el camino de convertirse en miembro de su sociedad en la que se interrelaciona y observa cómo los demás se interrelacionan entre sí, la lengua le sirve para mediatizar su relación en el intercambio de ideas, peticiones, órdenes, expresiones afectivas, etc. Parafraseando a Austin diríamos entonces que la lengua le sirve para hacer cosas. "The child's interpretation of the world is strongly pragmatic; she seeks knowledge in relation to her own interests, whether biological, social, or cognitive, and constructs new meanings therefrom" (Nelson, 2007: 12). El niño o la niña necesitará la lengua para hacer estas 
cosas, así que analizar la función que cumple resulta una tarea esencial para entender el desarrollo lingüístico infantil.

En el caso particular de los verbos, se ha insistido en la importancia de la pragmática en su adquisición, Rojas Nieto (2014) corrobora para el español lo mismo que Tomasello (1992) para el inglés: las combinaciones de verbo más su flexión se utilizan inicialmente en el discurso infantil con sus funciones pragmáticas primarias. Stoll (1998) argumenta también que la aparición de ciertas flexiones con determinados verbos se asocia a las funciones pragmáticas con las que se adquieren. Es por esta razón que resulta importante indagar qué tipo de función está cumpliendo en los inicios la lengua, como una primera ruta de exploración para entender su adquisición. ${ }^{1}$

\section{El gerundio}

El gerundio es una forma no personal del verbo, también llamada nominal o verboide cuya desinencia invariable es $-n d o$. Cuando el verbo con el que se conjuga pertenece a la primera conjugación esta terminación corresponde con -ando, ej.

\footnotetext{
${ }^{1}$ Así como en la literatura prevaleciente en adquisición del lenguaje, en este estudio se equipara el gerundio con otras formas verbales, porque, en primer lugar, se conjuga a partir de una raíz verbal. Y en segundo, aunque carece de persona, número o tiempo, tiene la característica de señalar, igual que otras formas verbales, el aspecto imperfectivo en español, con un significado ligado a duración o prolongación de las acciones. Las formas impersonales del verbo -infinitivo, gerundio y participio - se incluyen también en el estudio de la lingüística hispánica como parte de las formas verbales del español, como se puede apreciar en la obra de Moreno de Alba (1978).
} 
jugando. Cuando el verbo corresponde a la segunda y tercera conjugación termina en -endo, ej. leyendo, riendo. Históricamente se llamó no personal a esta forma puesto que no marca persona (lo mismo que sus contrapartes el participio y el infinitivo), pero carece además de número, modo y tiempo. Esta última significación la toma del verbo con el cual se construye.

El significado del gerundio es altamente dependiente no sólo de la naturaleza semántica del verbo con que se construye sino también del complejo oracional. Por ejemplo, se le reconoce un carácter durativo en la construcción con estar con verbos imperfectivos 'está comiendo' y un significado repetitivo con verbos perfectivos 'estuvo saltando' (RAE, 1986).

La noción también puede ser concebida como un proceso o una acción en progreso por lo que en inglés al morfema -ing equivalente al gerundio español se le llama progresivo.

Es común encontrar el gerundio en construcciones perifrásticas en las que forma una sola unidad con un verbo auxiliar, ej. La niña está jugando con los trenes en su cuarto vs. La niña está en su cuarto jugando con los trenes. La unidad perifrástica se reconoce porque en el segundo ejemplo el verbo "estar" lleva sus propios complementos, mientras que en el primero el sintagma "en su cuarto" no complementa a "estar" sino a "está jugando". Puesto que estar es verbo auxiliar y no verbo nuclear de carácter locativo se puede decir que se trata de una perífrasis verbal (Gómez Torrego, 1988: 136).

La perífrasis verbal con estar designa actos, acciones, eventos, etc., en desarrollo que coinciden con el momento de la enunciación, ej. La epidemia está empeorando (Maldo- 
nado, 2005: 434). En Maldonado (1999) se sugiere que para referir el punto crítico en que sucede el cambio de estado se emplea el marcador medio "se". Ej. Elia se está levantando. Asimismo, frente a una visión dinámica del significado del gerundio propone que se usa de igual manera para referir procesos en su momento estático, enfocados en el inicio del proceso. Esta construcción perifrástica es la más común en el habla (Luna Traill, 1991), y el característico significado aspectual imperfectivo del gerundio, que indica acción duradera, se intensifica puesto que se combina el significado aspectual imperfecto del verbo auxiliar "estar" con la imperfectividad propia del gerundio. Este carácter imperfectivo del gerundio se proyecta a la mayoría de las perífrasis, por ejemplo con: seguir, ir, venir, andar y llevar. Si el auxiliar es un verbo de movimiento (ir, venir o andar, parcialmente también llevar) + gerundio "se potencia aún más, aportándose así imágenes prolongativas de la acción dentro de una clara perspectiva estilística. No obstante, en las semiperífrasis empezar y acabar + gerundio domina el modo de acción de los primeros, la primera es incoativa y la segunda terminativa" (Gómez Torrego, 1988: 135). En el Esbozo (1986) se asigna en general un significado durativo a la construcción con estar y un carácter repetitivo cuando se combina con verbos perfectivos.

En su carácter aspectual se opone al participio, forma claramente perfectiva. El gerundio presenta una tensión media (la acción verbal ya se encuentra en curso) y distención media (aún queda aparte de la acción por desarrollar), frente al infinitivo, que presenta tensión máxima (queda toda la acción por desarrollar y distención cero; y el 
participio, que vienen configurado con tensión cero (la acción ya se ha desarrollado: no queda nada por desarrollar) y distención máxima (Alarcos Llorach, 1970: 57).

Roca Pons (1960) opone el presente de indicativo dentro de su dimensión aspectual imperfectiva por su significado habitual a la referencia deíctica del gerundio. Sin hacer mención al aspecto, De la Peña (1955) opone el gerundio con respecto al significado también habitual del infinitivo. Sin embargo, como el mismo De la Peña menciona, el gerundio también puede denotar un hecho que se verifica habitualmente en combinación con, por ejemplo, "vivir": viviendo sanamente.

En esencia, el gerundio refiere un proceso, con enfoque dinámico o estático con un valor aspectual. Si el verbo auxiliar de la construcción está en presente, entonces el gerundio tendrá un anclaje deíctico.

Este verboide tiene una funcion muy específica en el español, es durativa y de anclaje deíctico. Este anclaje podría facilitar su adquisición. Tomemos en cuenta, por otro lado, que dentro de su dimensión aspectual compite con el presente de indicativo, de modo que su especificidad frente a acciones durativas del aquí y el ahora dependerá del uso en la interacción social.

\section{El gerundio en la adquisición del lenguaje}

Mucho se ha discutido sobre la adquisición de la flexión verbal con la que los niños adquieren los verbos de acuerdo 
con el modo de acción verbal (Aktionsart) de estos. Existen numerosos estudios ya clásicos cuyo resultado generó lo que se llama la Hipótesis de la Congruencia Semántica: aspecto y flexión (Antinucci y Miller, 1976). Para el caso concreto de los progresivos la hipótesis señala que aparecen en el proceso de adquisición del lenguaje con verbos de actividad (de acuerdo con la clasificación de Vendler, 1967) y se extienden posteriormente a verbos de resultado y logro. El orden sería, por ejemplo, primero: jugando, barriendo, comiendo, durmiendo; y en segundo: construyendo, chocando, alcanzando, llegando, reconociendo, terminando. A este respecto varios estudios confirman esta hipótesis. Behrend, Harris y Cartwright (1995) encontraron una asociación entre el progresivo -ing con verbos de acción (running) y el pretérito -ed con verbos de resultado (reached), en los datos de Tomasello (1992) se documenta una alta frecuencia de verbos de acción asociados al progresivo. De igual manera, Li y Bowerman (1998) encuentran que los niños más pequeños producen los verbos atélicos con marcadores de progresivo. Mientras que algunos atribuyen a esto una explicación de tipo innatista (Bickerton, 1984), otros investigadores explican este fenómeno como una característica del contexto: Li y Bowerman argumentan que se debe a una característica particular de la lengua a un nivel básico de comunicación. Shirai y Anderson (1995) proponen que el fenómeno responde a la influencia del input que muestra una distribución sesgada hacia ese tipo de combinaciones, lo que llaman precisamente Hipótesis de la Distribución Sesgada. Sabine Stoll (1998), quien estudió este fenómeno en el ruso, lengua con morfología verbal asociada obligato- 
riamente al aspecto, propone que este fenómeno depende del contexto pragmático en que toma lugar el proceso de adquisición.

Para el inglés, la lengua más estudiada en el campo de la adquisición del lenguaje, se ha documentado que el progresivo -ing es el primer morfema productivo en el desarrollo de la adquisición temprana de esta lengua (Brown, 1973). Brown documentó además que los niños lo usan inicialmente en su forma "primitiva", sin el auxiliar am, is o are, incluso hasta lo que él llama el estadio V. Tomasello (1992) documentó que los verbos en progresivo son los únicos que se incorporan en contextos ostensivos. Ibbotson, Lieven y Tomasello (2013) se enfocaron en la relación de la flexión del gerundio y su significado en el contexto de la enunciación, en su estudio hacen patente que en el Habla Dirigida al Niño (HDN), a mayor duración de la actividad de la niña, mayor la probabilidad de dirigirse a ella con un verbo con terminación -ing. Esto explicaría el hecho de que el progresivo sea la primera flexión en la adquisición del inglés, ya que el aprendizaje está asociado con la percepción y la acción en el aquí y el ahora de la experiencia del niño.

\section{Los gerundios en la adquisición del español}

Hasta donde hemos logrado indagar, no existe ningún estudio que se centre directamente en la adquisición del gerundio en español. Sin embargo, ha sido mencionado por algunos autores en la búsqueda de la adquisición tanto de las flexiones como de las perífrasis verbales. Vincu- 
lado al estudio de la asociación entre flexiones y modo de acción de verbo (Aktionsart: véase supra). El estudio de Jackson-Maldonado y Maldonado (2002) documenta que el gerundio está asociado a verbos de acción, pero que es de aparición tardía. En el estudio de las perífrasis verbales con estar Ponce (2008) documenta, asimismo, que las perífrasis aspectuales "estar + gerundio" son de baja frecuencia y de aparición tardía y son usadas por los niños para hacer mención a actividades cotidianas. Mueller, Sebastián y Soto (1999) hicieron patente que la adquisición de las flexiones en español no se da de manera abstracta, sino fragmentaria, un verbo determinando aparece con una flexión determinada, y estas están asociadas tanto a la frecuencia del input materno como a estructuras de menor complejidad lingüística. Según sus observaciones, Juan (1;09,05-2;01,13), uno de los sujetos de su estudio, no utiliza en este periodo ningún tipo de gerundio, mientras que la otra, María $(1 ; 06,03$ $2 ; 06,16)$, presenta una sola emisión de gerundio a la edad de $2 ; 01,9$, y no es sino hasta los 2;03,11 que incrementa el uso a 6 ocurrencias (edad 2;04,22), luego a 7 emisiones $(2 ; 05,19)$ y 8 a los 2;06, 16. La frecuencia de uso de los gerundios no corresponde a la frecuencia del input, ya que la mamá de Juan usa gerundios con frecuencia, al contrario de la mamá de María. Lo que sugiere que el uso corresponde a la edad. En la niña estudiada por Rojas Nieto (2003) el gerundio es una forma poco frecuente en la madre y en la hija, de hecho, la niña comienza a usarlo hasta los 2;03 años. En otro estudio, Rojas Nieto (2011) documenta que las flexiones verbales se usan incluso con la función pragmática con la que son aprendidas. Por ejemplo Pres.2.sg aparece con peticiones in- 
terrogativas: “ime das?”. Las secuencias dialógicas aportan un apoyo importante para la formación del desarrollo de los paradigmas verbales (Rojas Nieto, 2014).

De acuerdo con estos estudios podríamos decir entonces que los gerundios del español son poco frecuentes en el habla infantil, de aparición tardía, y que esto no corresponde necesariamente a la frecuencia en el input materno.

\section{Pregunta de investigación}

El fin último de la lengua es la comunicación y el desarrollo de la lengua materna toma lugar en el seno de la interacción humana, esto implica que el infante convive día a día con la lengua como una herramienta para consolidar sus relaciones afectivas, sus deseos, sus necesidades. Por tanto resulta relevante preguntarse, ¿con qué funciones pragmáticas aparece el uso del gerundio en el proceso de la adquisición? Puesto que está en competencia con otras formas verbales es probable que se trate de un contexto con una función muy específica que relega su uso en la vida infantil a etapas tardías. ¿Cuáles son, pues, esas funciones? Esta pregunta es el hilo conductor de esta investigación.

\section{Descripción de la metodología}

Los datos analizados en este trabajo provienen de registros naturalistas de corte longitudinal recogidos en la base de datos ETAL-UnAM (Rojas, 2007). Para este estudio se eligieron 
tres niños: dos niñas y un niño del corpus. Los niños pertenecen a un grupo socioeoeconómico medio de clase educada de la Ciudad de México. Estos fueron grabados durante dos horas al día con intervalos aproximados de un mes en interacción natural con los miembros de su familia y en menor medida también en interacción con la observadora. Las dos niñas estudiadas, Elia y Flor, son hijas únicas, y Julio es el segundo de tres hermanos. En el ámbito sociocultural al que pertenecen estos tres niños, el Habla Dirigida al Niño (HDN) es parte de la interacción natural.

Se extrajeron todos los gerundios emitidos por los niños en su contexto inmediato de enunciación. Cada gerundio fue interpretado según la función pragmática con la que fue emitido por el niño. La muestra elegida suma un total de 96 horas. El número total de gerundios encontrados, así como la edad en que se registra el primer gerundio para cada sujeto puede verse en el cuadro 1 .

Cuadro 1. Presentación de los datos

\begin{tabular}{|l|c|c|c|c|c|}
\hline Nombre & $\begin{array}{c}\text { Horas } \\
\text { estudiadas }\end{array}$ & $\begin{array}{c}\text { Número de } \\
\text { gerundios } \\
\text { encontrados }\end{array}$ & $\begin{array}{c}\text { Edad del } \\
\text { primer } \\
\text { registro de } \\
\text { gerundio }\end{array}$ & LME & $\begin{array}{c}\text { Edad de la } \\
\text { última toma } \\
\text { documentada } \\
\text { en ETAL }\end{array}$ \\
\hline Elia & 32 & 103 & $1 ; 11,27$ & 2,06 & $4 ; 02,10$ \\
\hline Flor & 44 & 223 & $1 ; 10,24$ & 2,07 & $4 ; 00,2$ \\
\hline Julio & 20 & 95 & $2 ; 08,26$ & 2,1 & $3 ; 11,21$ \\
\hline
\end{tabular}

Para el análisis sucesivo se descartaron repeticiones o reformulaciones retomadas del discurso adulto, como las que se muestran en los ejemplos a continuación: 
Ejemplo de repetición que se ofrece como modelo en el discurso adulto

\section{Ejemplo 1}

Elia $(1 ; 11,2)$

Mirando una escena exterior

MAM: Sí ¿Qué están haciendo?

\section{jugando}

ELI: áno (jugando)

MAM: ajá, parece periquito, repite las finales, parece eco eco

Tanto las repeticiones como las reformulaciones constituyen en buena medida los gerundios incipientes, encontrados principalmente en el caso de Elia y Flor.

Ejemplo de reformulación

Ejemplo 2

Flor $(2 ; 2,20)$

Se escuchan martillazos

ABA: está arreglando las puertas el carpintero (afirmación) FLO: mjm, e capitero stá regando as cuetas

En ambos ejemplos podemos observar que se trata de una recuperación del discurso adulto. Aunque este tipo de intervención infantil juega un papel importante en el proceso de la adquisición de la lengua materna, y una función interlocutiva no discursiva ${ }^{2}$ que podría tratarse como la expresión de un acuerdo con la aseveración adulta, ${ }^{3}$ no se consideraron relevantes para este estudio que se enfoca en los actos de habla.

\footnotetext{
${ }^{2}$ Agradezco este comentario a la doctora Cecilia Rojas Nieto.

${ }^{3}$ Especialmente en el ejemplo de Flor en la que aparece la interjección "mjm".
} 


\section{Resultados}

En las 96 horas transcritas se halló un número relativamente bajo de gerundios (en comparación con otras formas verbales) y se constató que aparecen tardíamente en el desarrollo individual de cada niño. Lo anterior concuerda con los datos presentados para el estudio de la adquisición de morfemas verbales por Rojas (2003), y en el estudio de perífrasis de Ponce (2008), quienes usaron una muestra parcialmente semejante de la misma base de datos ETAL. Para cotejar con otras fuentes, hacemos notar que estos hallazgos concuerdan también en este sentido con los de Müller, Sebastián y Soto (1999) y con los de Jackson-Maldonado y Maldonado (2002).

\section{Las funciones del gerundio: actos de habla}

En lo que respecta a las funciones pragmáticas de cada emisión de gerundio, encontramos que los primeros contextos en que aparece en el habla infantil es a través de descripciones escuetas - sin que medien otras posibles funciones asociadas - de actividades que realizan personas, objetos o imágenes de libros o televisivas (véase cuadro 2). Llamamos descripción cuando el referente está anclado en el contexto deíctico de la enunciación, y el objeto/persona descrita está presente, llamamos narración cuando no lo está y conlleva temporalidad diferida. En el caso de Elia, la frecuencia más alta de estas descripciones está motivada por las preguntas adultas. En general, el porcentaje de res- 
puesta a preguntas adultas, especialmente formuladas con qué + hacer (Qué haces, qué hace) está al menos en los tres usos más frecuentes para los tres niños.

Cuadro 2. Funciones pragmáticas infantiles en el uso de gerundios

\begin{tabular}{|c|c|c|c|c|c|c|}
\hline \multirow{2}{*}{ Funciones } & \multicolumn{2}{|c|}{ Julio } & \multicolumn{2}{|c|}{ Elia } & \multicolumn{2}{|c|}{ Flor } \\
\hline & No. & $\%$ & No. & $\%$ & No & $\%$ \\
\hline Respuesta a pregunta & 15 & .21 & 31 & .32 & 33 & .20 \\
\hline $\mathrm{R}=$ Qué + Hacer/estar haciendo & 11 & .154 & 12 & .126 & 25 & .118 \\
\hline $\mathrm{R}=$ Otras preguntas & 4 & .056 & 19 & .2 & 8 & .037 \\
\hline Descriptiva & 33 & .47 & 26 & .27 & 63 & .40 \\
\hline Describe actividad propia & 19 & .267 & 9 & .094 & 30 & .142 \\
\hline Descripción actividad de otro(s) & 9 & .126 & 4 & .042 & 21 & .099 \\
\hline Descripción objetos & 4 & .056 & 8 & .084 & 6 & .028 \\
\hline Descripción (imagen) & 1 & .014 & 5 & .052 & 6 & .028 \\
\hline Narrativa & 3 & .04 & 6 & .06 & 8 & .05 \\
\hline Primera persona & 3 & .04 & 1 & .01 & 1 & .006 \\
\hline Segunda persona & - & - & - & 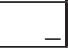 & 1 & .006 \\
\hline Tercera persona & - & - & 1 & .01 & 4 & .025 \\
\hline Plural & - & - & - & - & 2 & .012 \\
\hline Cuento & - & _ & 4 & .04 & _- & _ \\
\hline Concomitantes & 20 & .28 & 33 & .39 & 53 & .33 \\
\hline Indaga/Interroga & 5 & .070 & 1 & .01 & 6 & .028 \\
\hline Explica & 1 & .014 & 6 & .063 & 5 & .023 \\
\hline Justifica & - & - & 8 & .084 & 5 & .023 \\
\hline Acusa & 1 & .014 & - & 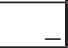 & 7 & .033 \\
\hline Ordena & 2 & .028 & 1 & .01 & 6 & .028 \\
\hline Avisa & 2 & .028 & - & - & 5 & .023 \\
\hline Otras & 9 & .126 & 17 & .189 & 19 & .09 \\
\hline Total & 71 & 1 & 96 & 1 & 157 & 1 \\
\hline
\end{tabular}

$\mathrm{R}=$ respuesta $\mathrm{a}$

Con el fin de hacer más explícitas las funciones del cuadro 2 se presentan a continuación ejemplos de cada una. 


\section{Función de respuesta a pregunta}

Un ejemplo común en el contexto de la respuesta a preguntas adultas lo encontramos precisamente en la interacción de lectura de libros, véase el siguiente ejemplo:

Pregunta más frecuente: Descripción en rutina de libros Ejemplo 3

Elia $(4 ; 00)$

Con su papá, mirando un libro

ELI: y ésta es una niña y éste es un borrego

PAP: ¿y qué está haciendo la niña?

ELI: durmiendo

PAP: durmiendo... pero, a ver, fíjate se está durmiendo y qué...

Otro ejemplo representativo de las preguntas adultas formuladas que dan como resultado una respuesta con gerundio se encuentra en el quehacer cotidiano del niño, en la que el adulto hace una pregunta sobre su actividad por resultar sospechosa o extraña, en este ejemplo la madre no está atendiendo la actividad del niño y sospecha que éste se encuentra haciendo algo indebido.

Ejemplo 4

Julio $(3 ; 03,04)$

MAM: ¿qué estás haciendo? (afuera del cuarto)

OBS: pobrecita.

JUL: (sonidos de disparo)

JUL: _ _ tuca cupada. 
OBS: estás ocupada.

MAM: ¿qué estás haciendo? (entrando)

JUL: jugando con las pistola.

MAM: ay pero me diste un balazo en la panza.

O bien, un ejemplo interesante lo encontramos en la rutina del saludo.

Ejemplo 5

Flor $(3 ; 02,20)$

La mamá de Flor llega y saluda

MAM: hola

FLOR: hola

MAM: ¿qué haces?

FLOR: chiflando

MAM: ¿qué chiflas?

\section{Función descriptiva}

Actividad propia (rutina de saludo)

Ejemplo 6

Flor $(2 ; 02,20)$

Llega la mamá de Flor.

OBS: ¿quiúbo Nida? (mamá de Flor)

MAM: hola (llega)

FLOR: etó comiendo

Esta rutina nos parece notable porque tenemos la evidencia de que al volverse cotidiana, la niña responde al saludo narrando su propia actividad incluso sin que el adulto formule la pregunta. Estas construcciones interrogativas 
conllevan implícita la petición de una descripción sobre la actividad. En cuanto a las respuestas que aparecen como una construcción verbal con gerundio después de una interrogativa adulta formulada con la perífrasis con estar (ejemplo $3)$, pueden considerarse el par adyacente dentro del discurso. Este fenómeno merece una mención aparte cuando se trata de una respuesta a gerundio formulada con Qué + hacer respondida por la niña con un gerundio aislado. Una posible explicación podría ser, como en los casos presentados en los ejemplos 5 y 6 , describir la propia actividad después de que el saludo se ha rutinizado. Por otro lado, puesto que el presente indicativo puede expresar un valor actual durativo (Moreno de Alba, 1978), la encontramos en algunos ejemplos usada de manera indiferente por los adultos, incluso adyacente dentro del mismo enunciado: “¿Qué haces?” “QQué estás haciendo?” Es posible que los niños estén reconociendo el valor descriptivo del gerundio al responder a una interrogativa formulada sin el auxiliar estar. Un estudio más enfocado en este fenómeno podría corroborarnos el porqué de este fenómeno.

En Elia el uso de gerundios es particularmente frecuente como respuesta a otras preguntas como "¿qué pasa?", aunque no se repiten como tipo, de la manera en que resulta frecuente la combinación Qué + hacer:

Ejemplo 7

Elia $(3 ; 02,26)$

La niña Elia juega con una muñeca

(Extiende la cobija en el aire y tapa a la muñeca con ella y la carga).

ELI: Llévala... ¡uy! juy! ¿señor? 
(Elia sale de su cuarto y camina por el pasillo).

\section{PAP: ¿Qué pasa?, ¿qué pasa? \\ ELI: $\quad$ Se está murieno.}

Un ejemplo como el anterior fue clasificado dentro del rubro descripción actividad propia, la función más frecuente en Julio y Flor, y la tercera en Elia. Tanto en Flor como en Julio son también frecuentes contextos en que los gerundios son usados para describir la actividad del otro. A continuación presento un ejemplo:

Descripción actividad de otro

Ejemplo 8

Flor $(4 ; 00)$

COM: Flor mira a la ventana

FLOR: está cogiendo su escoba el niño

ABA: ¿Sí? Sí pero eso no es peligroso, lo peligroso era el machete

Los niños usan el gerundio también para describir objetos (con frecuencia juguetes) como en el ejemplo siguiente:

Descripción de objetos

Ejemplo 9

Julio $(3 ; 07,24)$

Jugando con LEGOS ${ }^{\circledast}$

JUL: sí, güela...

OBS: vuela-vuela (cantando)

JUL: ¡no! éste está vuelando

OBS: ¿está vuelando?

JUL: sí

HNO: tiene una manera de hacerlo volar 


\section{Concomitancia de funciones}

Encontramos también interrogaciones infantiles que pueden estar representando el discurso adulto en la voz del niño:

Ejemplo 10

Julio $(3 ; 06,25)$

Julio mira por la cámara

JUL: ¿qué está haciendo Emilio (hermano)?

OBS: tú dime qué hace, no veo

Otras funciones más especializadas fueron también encontradas en los tres niños. ${ }^{4}$ Aunque sigue tratándose de descripciones, estas cumplen, a su vez, otras funciones más especializadas que pueden estar correspondiendo tanto a las necesidades individuales del entorno de cada niño como a la reproducción de los modelos de los individuos que los rodean, ya sea los adultos o los hermanos mayores. Las tres más importantes (o al menos más frecuentes de la base de datos) son funciones para dar alguna explicación, Julio $(3 ; 04,11)$ : tocotorreando (estoy cotorreando); justificar, Elia $(2 ; 08,25):$ odita pédate toy jugando (ahorita, espérate, estoy jugando); acusar, Flor $(2 ; 07,22)$ meta ensuciando mi papá con su pie (me está ensuciando mi papá con su pie); y avisar, Flor $(2 ; 07,22)$ metoy haciendo popó (me estoy haciendo popó). Es importante tomar en cuenta que si bien se trata de

\footnotetext{
${ }^{4}$ Cuando apareció una frecuencia mayor a 5 emisiones en alguno de ellos, las consideramos de manera independiente para el análisis, pero cuando ninguno de ellos tenía al menos 5 emisiones de esa función se descartó del conteo individual y se acumularon dentro del rubro "Otras" del cuadro 2.
} 
oraciones que contienen un gerundio se asocian en general a un desarrollo global de la lengua, lo que permite también que los significados sean más complejos. En Elia, las tres primeras funciones documentadas, en 2;05 - 2;06, son las respuestas a preguntas y descripción de objeto e imagen. En Julio, entre los 2;08 y 3;03, descripción de un tercero, interroga y describe actividad propia y, finalmente, Flor, 1;11,27 - 2;01,18, describe actividad propia, responde a pregunta y describe imagen. Esto nos hace pensar que la mera descripción es la función más básica, y que poco a poco va especializándose hacia otras funciones pragmáticas que permiten a los niños desarrollarse en su medio social mediante a la referencia durativa de una acción anclada en el momento de la enunciación.

Por último, se presentan los cinco primeros enunciados con gerundio de cada uno de los niños:

Cuadro 3. Los cinco primero enunciados con gerundio en cada niño

\begin{tabular}{|l|l|l|}
\hline Julio & Elia & Flor \\
\hline $\begin{array}{l}\mathrm{R}=\text { pintando um Fabián } \\
(2 ; 08)\end{array}$ & $\begin{array}{l}\mathrm{R}=\text { Ta badiendo } \\
(2 ; 05,15)\end{array}$ & $\begin{array}{l}\mathrm{D}-\mathrm{AP}=\text { oy viendo mamá } \\
\text { (en el espejo) }(1 ; 10,24)\end{array}$ \\
\hline $\mathrm{P}=$ ta haciendo? $(2 ; 11)$ & $\begin{array}{l}\mathrm{D}-\mathrm{IL}=\text { ta mañando } \\
(2 ; 06,22)\end{array}$ & $\begin{array}{l}\mathrm{R}=\text { pam pomiendo } \\
(2 ; 01,04)\end{array}$ \\
\hline $\begin{array}{l}\mathrm{D}-\mathrm{AP}=\text { echano agua } \\
(3 ; 00)\end{array}$ & $\begin{array}{l}\mathrm{D}-\mathrm{O}=\text { ta bañaño } \\
(2 ; 06,22)\end{array}$ & $\begin{array}{l}\mathrm{R}=\text { jugando a la tzeda } \\
\text { (tierra) }(2 ; 01,04)\end{array}$ \\
\hline $\begin{array}{l}\mathrm{D}-\mathrm{AP}=\text { tu jugando } \\
\text { mitendo }(3 ; 03)\end{array}$ & $\begin{array}{l}\mathrm{R}=\text { no, ta bincando } \\
(2 ; 06,22)\end{array}$ & $\begin{array}{l}\mathrm{R}=\text { tirando basura } \\
(2 ; 01,18)\end{array}$ \\
\hline $\begin{array}{l}\mathrm{D}-\mathrm{O}=\text { ta esculniendo } \\
(3 ; 03)\end{array}$ & $\begin{array}{l}\mathrm{R}=\text { Tomiendo su leche } \\
(3 ; 03)\end{array}$ & $\begin{array}{l}\mathrm{D}-\mathrm{IL}=\text { un pedo (perro) } \\
\text { nadando } \\
(2 ; 01,18)\end{array}$ \\
\hline
\end{tabular}

$\mathrm{R}=$ Respuesta, $\mathrm{P}=$ Pregunta, $\mathrm{D}-\mathrm{AP}=$ Descripción de actividad propia, $\mathrm{D}-\mathrm{O}=$ Descripción de objeto, D-IL= Descripción de imagen-libro. 
La información presentada en el cuadro 3 nos permite apreciar que las estructuras enunciativas más tempranas constituyen meras descripciones, impulsadas primordialmente por las preguntas adultas (33\%) frente a la función descriptiva de la propia actividad (20\%), la segunda más frecuente en este cuadro y la más frecuente dentro de las subfunciones descriptivas en el cuadro 2. En segundo lugar se encuentran en igual medida tanto las descripciones de objetos como de imágenes (13\%). Cabe preguntarse si este orden en la preferencia infantil está promovido por las preguntas adultas, que enfocan la atención primordialmente hacia la descripción de su propia actividad. Esta respuesta queda pendiente para una futura investigación.

\section{Discusión}

Dados estos resultados, que nos indican que los gerundios se adquieren en un contexto temprano en los que describir el acontecer es prácticamente el único fin, parece relevante considerar algunos estudios sobre el gerundio en la narrativa infantil. A este respecto, se revisó la investigación en la que Berman, Slobin y otros colaboradores exponían a los niños a un famoso cuento ilustrado que habla de un niño al que se le pierde su rana. Berman y Slobin analizaron la distribución del progresivo inglés -ing en todos los enunciados que contenían un verbo principal explícito (57\% en los niños de 3 años en comparación con el 80\% en niños de 4 y 5 años). Descubrieron que los niños menores usan el aspecto progresivo como no marcado, como una forma básica 
de describir eventos que se construyen como concernientes al momento del habla, "This claim is supported by occasional 3- and 4-year old extensions of progressive forms from process verbs to stative verbs which typically disallow their use" (Berman y Slobin, 1994: 139). En este mismo volumen, Sebastián y Slobin (1994) descubrieron para el español que el uso del gerundio es mucho más frecuente en los niños de 3 y 4 años que en los mayores (entre 5 y 9) e incluso los adultos. Atribuyen a este fenómeno precisamente la función descriptiva del gerundio. Esto, aunado a que les permite indicar traslapes temporales en la narración de la historia, puesto que aún no utilizan oraciones subordinadas y otros elementos léxicos que indiquen traslape temporal. Los niños preescolares $(4 ; 00$ a 5;00) refieren las situaciones icónicas como durativas mediante el uso del gerundio independiente, para contrastarlo con las no durativas, ej. de un niño de 4;07: $Y$ aquí se sentó en el agua y el perro subiéndose a su cabeza (Sebastián y Slobin, 1994: 248). Según los autores, esto indica que los niños utilizan el gerundio como un marcador de duración.

Podríamos decir entonces que estos datos apoyan nuestros resultados de investigación, pues refuerzan el carácter primario descriptivo de los gerundios.

Como documentamos arriba, otros autores han mencionado la asociación que existe en la adquisición de los verbos infantiles en relación con su función pragmática. La manifestación lingüística aquí estudiada no es precisamente considerada como un verbo, sino como un verboide o forma no personal/nominal del verbo. Su significado global depende fuertemente no sólo del verbo con el que se combina sino 
de todo el complejo oracional. Su significado puede ser durativo, prolongativo, dinámico, estático, etc. con enfoque al inicio, en medio o final del proceso referido, pero se trata siempre del enfoque de una acción en progreso, un proceso. Atendiendo a esta reflexión no resulta extraño que, según nos lo revelan nuestros datos, su función dentro de los actos de habla corresponde a la función asertiva: una descripción que está impulsada en gran medida por la pregunta del adulto y el uso mismo que este hace para comunicarse con el niño. Cabe mencionar a este respecto que Rakoczy y Tomasello (2009) documentan que los niños angloparlantes no realizan tareas de comprensión con función asertiva con éxito sino hasta los 3 años. Según nuestros resultados, y tomando en cuenta que este estudio comprende sólo análisis de producción, esto no parece ser cierto también para los niños hispanohablantes; convendría realizar un estudio de comprensión de las funciones asertivas en la adquisición temprana del español para saber si existe una diferencia con el inglés a este respecto.

En el estudio de la adquisición temprana del gerundio, resulta relevante cuestionar su carácter deíctico, este fenómeno de la lengua permite al infante vincular la lengua con el mundo (Tanz, 1980). Frente a los estudios de Tomasello y Kruger (1992) sobre adquisición verbal en contextos ostensivos, llama la atención que los verbos en progresivo del inglés sean los únicos adquiridos precisamente en estos contextos, quizás en este sentido sea lógico considerar que su primer uso infantil sea la descripción de un referente presente. El gerundio del español, al contrario que el progresivo del inglés, es de aparición tardía, es posible entonces que 
esto se deba a que en español compite con otras formas verbales, la pregunta indiferente qué haces/qué estás haciendo sugiere que compite con el presente indicativo, competencia de formas que ya había notado Roca Pons (1960).

La adquisición del gerundio requiere una representación mental de un proceso durativo, con anclaje deíctico (dependiente también de la flexión temporal del verbo auxiliar) que, según demuestran los datos, se adquiere en un contexto que exige la descripción de actividades y se especializa rápidamente para realizar una función más específica dentro de la lengua.

\section{Bibliografía}

Alarcos Llorach, E. (1970), Estudios de gramática funcional del español, Madrid, Gredos.

Antinucci, F. y R. Miller (1976), "How children talk about what happened", Journal of Child Language, 3, pp. 167-189.

Behrend D. A., L. L. Harris y K. B. Cartwright (1995), "Morphological cues to verb meaning: verb inflections and the initial mapping of verb meanings", 22 pp. 89106.

Berman, R. A. y D. I. Slobin (1994), Relating events in narrative. A crosslinguistic developmental study, Hillsdale, NJ, Lawrence Erlbaum.

Bickerton, D. (1984), “The Languagebioprogram hypothesis", The Behavioral and Brain Sciences, 7, pp. 173-188. 
Brown, R. (1973), A first language: the early stages, Oxford, Harvard University Press.

Gómez Torrego, L. (1988), Perífrasis verbales, Madrid, Arco/Libros.

Ibbotson, P., E. Lieven y M. Tomasello (2013), “The communicative contexts of grammatical aspect use in English", Journal of Child Language, 40, pp. 1-19.

Jackson-Maldonado, D. y R. Maldonado (2002), “Determinaciones semánticas de la flexión verbal en la adquisición del español", en C. Rojas Nieto y L. de León (eds.), La adquisición de la lengua materna, México, Universidad Nacional Autónoma de México-Centro de Investigaciones y Estudios Superiores en Antropología Social.

LI, P. y M. BowERMAN (1998), "The acquisition of lexical and grammatical aspect in Chinese", First Language, 18, pp. 311-350.

Luna Traill, E. (1991), Sintaxis de los verboides en el habla culta de la Ciudad de México, México, Universidad Nacional Autónoma de México.

Maldonado R. (1999), A media voz: problemas conceptuales del clítico se en español, México, Universidad Nacional Autónoma de México.

(2005), "El gerundio español como progresivo estático. Análisis cognoscitivo", Revista Española de Lingüística, 35, pp. 433-460.

Moreno de AlBA, J. G. (1978), Valores de las formas verbales en el español de México, México, Universidad Nacional Autónoma de México.

Mueller Gathercole, V. C., E. Sebastián y P. Soto (1999), “The early acquisition of Spanish verbal mor- 
phology: across-the-board International", Journal of Bilingualism, 3, pp. 133-182.

Nelson, K. (2007), Young minds in social worlds: experience, meaning, and memory, Cambridge, MA, Harvard University Press.

Peña, A. De la (1955), Tratado del gerundio, México, Jus.

Ponce Romero, X. (2008), Adquisición temprana de los verbos ser $y$ estar en español, tesis de licenciatura, México, Universidad Nacional Autónoma de México.

Rakoczy, H. y M. Tomasello (2009), "Done wrong or said wrong? Young children understand the normative directions of fit of different speech acts", Cognition, 113, pp. 205-212.

Real Academia Española (1986), Esbozo de una nueva gramática de la lengua española, Madrid, Espasa-Calpe.

Roca-Pons, J. (1960), Introducción a la gramática, Barcelona, Teide.

Rojas Nieto, C. (2003), "Early acquisition of Spanish verb inflection: a usage-based account", Psychology of Language and Communication, 7, pp. 17-36. (2007), "La base de datos ETAL. Etapas tempranas en la adquisición del lenguaje", Jornadas Filológicas 2005. Memoria, México, Universidad Nacional Autónoma de México, pp. 575-599.

(2011), "Developing first contrasts in Spanish verb inflection: usage and inflection", en I. Arnon y E. V. Clark (eds.), Experience, variation and generalization. Learning a first languaje, Ámsterdam/Filadelfia, John Benjamins, pp. 53-72. 
Rojas Nieto, C. (2014), "Verb sequences and early verb inflection in Spanish. Frequency, patterning and possible effects", Language, Interaction and Acquisition, 5:1, Ámsterdam/Filadelfia, John Benjamins, pp. 8-99.

Sebastián, E. y D. I. Slobin (1994), “Development of linguistic forms=spanish", en R. A. Berman y D. I. Slobin (eds.), Relating Events in Narrative. A Crosslinguistic developmental study, Hillsdale, NJ, Lawrence Erlbaum. Shirai, Y. y R. Anderson (1995), “The acquisition of tense/ aspect morphology: a prototype account", Language, 71, pp. 743-762.

STOLL, S. (1998), "The role of aktionsart in the acquisition of russian aspect", First Language, 18, pp. 351-378.

TANZ, C. (1980), Studies in the acquisition of deictic frames, Nueva York, Cambridge University Press.

Tomasello, M. (1992), First verbs. A case study of early grammatical development, Nueva York, Cambridge University Press.

_ ring verbs in ostensive and non-ostensive contexts", Journal of Child Language, 19, pp. 311-333.

Vendler, Z. (1967), Linguistics in philosophy, Ítaca, Nueva York, Cornell University Press. 